\title{
Remarks on contractive mappings via $\Omega$-distance
}

\author{
Leila Gholizadeh ${ }^{1}$ and Erdal Karapınar ${ }^{2 *}$
}

${ }^{*}$ Correspondence: erdalkarapinar@yahoo.com; ekarapinar@atilim.edu.tr ${ }^{2}$ Department of Mathematics, Atilim University, Incek, Ankara 06836, Turkey

Full list of author information is available at the end of the article

\begin{abstract}
Very recently, some authors discovered that some fixed point results in the context of a $G$-metric space can be derived from the fixed point results in the context of a quasi-metric space and hence the usual metric space. In this article, we investigate some fixed point results in the framework of a $G$-metric space via $\Omega$-distance that cannot be obtained by the usual fixed point results in the literature. We also add an application to illustrate our results.
\end{abstract}

MSC: $47 \mathrm{H} 10 ; 54 \mathrm{H} 25 ; 46 \mathrm{~J} 10 ; 46 \mathrm{J15}$

Keywords: $\Omega$-distance; fixed point; G-metric space

\section{Introduction and preliminaries}

Very recently, Jleli and Samet [1] and Samet et al. [2] proved that some fixed point results in the setting of G-metric spaces, introduced by Sims and Mustafa [3], are consequences of the well-known fixed point theorem in the context of the usual metric space. Indeed, authors in $[1,2]$ noticed that $G(x, y, y)=q(x, y)$ is a quasi-metric and obtained that the results are just a characterization of existence results in the framework of a quasi-metric. On the other hand, a G-metric was introduced as a generalization of the (usual) metric. Basically, $G$-metrics claim the geometry of three points instead of two points. Consequently, Jleli and Samet [1] and Samet et al. [2] concluded that if the expression in the fixed point theorem can be reduced to two points, then it can be written as a consequence of the related existence result in the literature.

Recently, Saadati et al. [4] introduced the concept of $\Omega$-distance on a complete $G$-metric space as a generalized notion of $\omega$-distance due to Kada et al. [5]. In these papers, the authors investigate the existence/uniqueness of a fixed point of certain operators in this setting. In this paper, we revise some published papers (see, e.g., [6, 7]) and improve the statements in a way that cannot be manipulated by the techniques used in $[1,2]$ (see also [8-10]).

We first recall some necessary definitions and basic results on the topics in the literature.

Definition 1 ([3]) Let $X$ be a non-empty set. A function $G: X \times X \times X \rightarrow[0, \infty)$ is called a $G$-metric if the following conditions are satisfied:

(i) $G(x, y, z)=0$ if $x=y=z$ (coincidence),

(ii) $G(x, x, y)>0$ for all $x, y \in X$, where $x \neq y$,

(iii) $G(x, x, z) \leq G(x, y, z)$ for all $x, y, z \in X$, with $z \neq y$,

(iv) $G(x, y, z)=G(p\{x, y, z\})$, where $p$ is a permutation of $x, y, z$ (symmetry), 
(v) $G(x, y, z) \leq G(x, a, a)+G(a, y, z)$ for all $x, y, z, a \in X$ (rectangle inequality).

A $G$-metric is said to be symmetric if $G(x, y, y)=G(y, x, x)$ for all $x, y \in X$.

Definition 2 ([3]) Suppose that $(X, G)$ is a $G$-metric space.

(1) A sequence $\left\{x_{n}\right\}$ in $X$ is said to be $G$-Cauchy sequence if, for each $\varepsilon>0$, there exists a positive integer $n_{0}$ such that for all $n, m, l \geq n_{0}, G\left(x_{n}, x_{m}, x_{l}\right)<\varepsilon$.

(2) A sequence $\left\{x_{n}\right\}$ in $X$ is said to be $G$-convergent to a point $x \in X$ if, for each $\varepsilon>0$, there exists a positive integer $n_{0}$ such that for all $m, n \geq n_{0}, G\left(x_{m}, x_{n}, x\right)<\varepsilon$.

Definition $3([4])$ Let $(X, G)$ be a $G$-metric space. Then a function $\Omega: X \times X \times X \rightarrow$ $[0, \infty)$ is called an $\Omega$-distance on $X$ if the following conditions are satisfied:

(a) $\Omega(x, y, z) \leq \Omega(x, a, a)+\Omega(a, y, z)$ for all $x, y, z, a \in X$,

(b) $\Omega(x, y, \cdot), \Omega(x, \cdot, y): X \rightarrow[0, \infty)$ are lower semi-continuous for any $x, y \in X$,

(c) for each $\varepsilon>0$, there exists $\delta>0$ such that $\Omega(x, a, a) \leq \delta$ and $\Omega(a, y, z) \leq \delta$ imply $G(x, y, z) \leq \varepsilon$.

Example $4([4])$ Suppose that $(X, d)$ is a metric space. Let $G: X^{3} \longrightarrow[0, \infty)$ be defined as follows:

$$
G(x, y, z)=\max \{d(x, y), d(y, z), d(x, z)\}
$$

for all $x, y, z \in X$. Then one can easily show that $\Omega=G$ is an $\Omega$-distance on $X$.

Example 5 ([4]) Let $X=\mathbb{R}$ and $(X, G)$ be a $G$-metric, where

$$
G(x, y, z)=\frac{1}{3}(|x-y|+|y-z|+|x-z|)
$$

for all $x, y, z \in X$. If we define $\Omega: \mathbb{R}^{3} \longrightarrow[0, \infty)$ as follows:

$$
\Omega(x, y, z)=\frac{1}{3}(|z-x|+|x-y|)
$$

for all $x, y, z \in X$, then it is an $\Omega$-distance on $\mathbb{R}$.

We refer, e.g., to $[4,11]$ for more details and examples on the topic.

Lemma 6 [4] Suppose that $(X, G)$ is a G-metric space and $\Omega$ is an $\Omega$-distance on $X$. Let $\left\{x_{n}\right\},\left\{y_{n}\right\}$ be sequences in $X$ and $\left\{\alpha_{n}\right\},\left\{\beta_{n}\right\}$ be sequences in $[0, \infty)$ converging to zero and $x, y, z, a \in X$. Then

(a) if $\Omega\left(y, x_{n}, x_{n}\right) \leq \alpha_{n}$ and $\Omega\left(x_{n}, y, z\right) \leq \beta_{n}$ for $n \in \mathbb{N}$, then $G(y, y, z)<\varepsilon$, and hence $y=z$;

(b) if $\Omega\left(y_{n}, x_{n}, x_{n}\right) \leq \alpha_{n}$ and $\Omega\left(x_{n}, y_{m}, z\right) \leq \beta_{n}$ for $m>n$, then $G\left(y_{n}, y_{m}, z\right) \rightarrow 0$, and hence $y_{n} \rightarrow z$

(c) if $\Omega\left(x_{n}, x_{m}, x_{l}\right) \leq \alpha_{n}$ for any $l, m, n \in \mathbb{N}$ with $n \leq m \leq l$, then $\left\{x_{n}\right\}$ is a G-Cauchy sequence;

(d) if $\Omega\left(x_{n}, a, a\right) \leq \alpha_{n}$ for any $n \in \mathbb{N}$, then $\left\{x_{n}\right\}$ is a G-Cauchy sequence.

Definition 7 ([4]) Suppose that $(X, G)$ is a $G$-metric space and $\Omega$ is an $\Omega$-distance on $X$. $(X, G)$ is called $\Omega$-bounded if there is a constant $C>0$ with $\Omega(x, y, z) \leq C$ for all $x, y, z \in X$. 
Definition 8 Let $(X, \leq)$ be a partially ordered set. A self-mapping $T: X \rightarrow X$ is said to be non-decreasing if, for $x, y \in X$,

$$
x \leq y \quad \Longrightarrow \quad T(x) \leq T(y)
$$

The tripled $(X, G, \leq)$ is called a partially ordered $G$-metric space if $(X, \leq)$ is a partially ordered set endowed with a $G$-metric on $X$; see also $[12,13]$.

\section{Fixed point theorems on partially ordered G-metric spaces}

We start this section with the following classes of mappings:

$$
\begin{aligned}
& \Phi=\{\phi \mid \phi:[0, \infty) \rightarrow[0, \infty) \text { continuous, non-decreasing }\} \text { and } \\
& \Psi=\{\psi \mid \psi:[0, \infty) \rightarrow[0, \infty) \text { continuous, non-decreasing }\}
\end{aligned}
$$

with $\phi^{-1}(\{0\})=\psi^{-1}(\{0\})=\{0\}$.

Definition 9 Let $(X, \leq)$ be a partially ordered space. Suppose that there exists a $G$-metric on $X$ such that $(X, G)$ is a complete $G$-metric space. A self-mapping $T: X \rightarrow X$ is said to be a generalized weak-contraction mapping if it satisfies the following condition:

$$
\psi\left(\Omega\left(T x, T^{2} x, T y\right)\right) \leq \psi(\Omega(x, T x, y))-\phi(\Omega(x, T x, y)) \quad \text { for all } x, y \in X, \text { with } x \leq y,
$$

where $\psi \in \Psi$ and $\phi \in \Phi$.

Theorem 10 Let $(X, G, \leq)$ be a partially ordered complete $G$-metric space, and let $\Omega$ be an $\Omega$-distance on $X$. Suppose that a non-decreasing self-mapping $T: X \rightarrow X$ is a generalized weak-contraction mapping, that is,

$$
\psi\left(\Omega\left(T x, T^{2} x, T y\right)\right) \leq \psi(\Omega(x, T x, y))-\phi(\Omega(x, T x, y)) \text { for all } x, y \in X \text {, with } x \leq T x,
$$

with $\psi \in \Psi$ and $\phi \in \Phi$. Suppose also that $\inf \{\Omega(x, y, x)+\Omega(x, y, T x)+\Omega(x, T x, y): x \leq T x\}>$ 0 for every $y \in X$ with $y \neq T y$. If there exists $x_{0} \in X$ with $x_{0} \leq T x_{0}$, then $T$ has a unique fixed point, say $u \in X$. Moreover, $\Omega(u, u, u)=0$.

Proof If $x_{0}=T x_{0}$, then the proof is finished. Suppose that $x_{0} \neq T x_{0}$. Since $x_{0} \leq T x_{0}$ and $T$ is non-decreasing, we obtain

$$
x_{0} \leq T x_{0} \leq T^{2} x_{0} \leq \cdots \leq T^{n+1} x_{0} \leq \cdots .
$$

Now, if for some $n \in \mathbb{N}, \Omega\left(T^{n} x_{0}, T^{n+1} x_{0}, T^{n+1} x_{0}\right)=0$, then

$$
\begin{aligned}
\psi\left(\Omega\left(T^{n+1} x_{0}, T^{n+2} x_{0}, T^{n+2} x_{0}\right)\right) \leq & \psi\left(\Omega\left(T^{n} x_{0}, T^{n+1} x_{0}, T^{n+1} x_{0}\right)\right) \\
& -\phi\left(\Omega\left(T^{n} x_{0}, T^{n+1} x_{0}, T^{n+1} x_{0}\right)\right),
\end{aligned}
$$

then $\Omega\left(T^{n+1} x_{0}, T^{n+2} x_{0}, T^{n+2} x_{0}\right)=0$. Due to [(a), Definition 3], we have $\Omega\left(T^{n} x_{0}, T^{n+2} x_{0}\right.$, $\left.T^{n+2} x_{0}\right)=0$. On the other hand, by [(c), Definition 3], we easily derive that $G\left(T^{n} x_{0}, T^{n+2} x_{0}\right.$, $\left.T^{n+2} x_{0}\right)=0$, which completes the proof. 
Consequently, throughout the proof, we suppose that $\Omega\left(T^{n} x_{0}, T^{n+1} x_{0}, T^{n+1} x_{0}\right)>0$ for all $n \in \mathbb{N}$. Hence, we have

$$
\begin{aligned}
\psi\left(\Omega\left(T^{n} x_{0}, T^{n+1} x_{0}, T^{n+1} x_{0}\right)\right) \leq & \psi\left(\Omega\left(T^{n-1} x_{0}, T^{n} x_{0}, T^{n} x_{0}\right)\right) \\
& -\phi\left(\Omega\left(T^{n-1} x_{0}, T^{n} x_{0}, T^{n} x_{0}\right)\right)
\end{aligned}
$$

which yields that

$$
\psi\left(\Omega\left(T^{n} x_{0}, T^{n+1} x_{0}, T^{n+1} x_{0}\right)\right) \leq \psi\left(\Omega\left(T^{n-1} x_{0}, T^{n} x_{0}, T^{n} x_{0}\right)\right) .
$$

As a result, we conclude that $\left\{\Omega\left(T^{n} x_{0}, T^{n+1} x_{0}, T^{n+1} x_{0}\right)\right\}$ is non-increasing. Thus, there exists $r \geq 0$ such that

$$
\lim _{n \rightarrow \infty} \Omega\left(T^{n} x_{0}, T^{n+1} x_{0}, T^{n+1} x_{0}\right)=r
$$

We shall show that $r=0$. Suppose, on the contrary, that $r>0$. Then we have $\phi(r)>0$. Letting $n \rightarrow \infty$ on (2.1), we obtain

$$
\psi(r) \leq \psi(r)-\phi(r)
$$

a contraction. Hence, we have

$$
\lim _{n \rightarrow \infty} \Omega\left(T^{n} x_{0}, T^{n+1} x_{0}, T^{n+1} x_{0}\right)=0
$$

Recursively, we obtain that

$$
\lim _{n \rightarrow \infty} \Omega\left(T^{n} x_{0}, T^{n+1} x_{0}, T^{n+t} x_{0}\right)=0
$$

for every $t \in \mathbb{N}$.

Let $l \geq m \geq n$ with $m=n+k$ and $l=m+t(k, t \in \mathbb{N})$. By the triangle inequality, we derive that

$$
\begin{aligned}
\Omega\left(T^{n} x_{0}, T^{m} x_{0}, T^{l} x_{0}\right) \leq & \Omega\left(T^{n} x_{0}, T^{n+1} x_{0}, T^{n+1} x_{0}\right)+\Omega\left(T^{n+1} x_{0}, T^{m} x_{0}, T^{l} x_{0}\right) \\
\leq & \Omega\left(T^{n} x_{0}, T^{n+1} x_{0}, T^{n+1} x_{0}\right)+\Omega\left(T^{n+1} x_{0}, T^{n+2} x_{0}, T^{n+2} x_{0}\right) \\
& +\cdots+\Omega\left(T^{m-1} x_{0}, T^{m} x_{0}, T^{l} x_{0}\right) .
\end{aligned}
$$

Letting $n \rightarrow \infty$ in the inequality above, by keeping the limits (2.2) and (2.3), we obtain

$$
\lim _{n, m, l \rightarrow \infty} \Omega\left(T^{n} x_{0}, T^{m} x_{0}, T^{l} x_{0}\right)=0
$$

Therefore, $\left\{T^{n} x_{0}\right\}$ is a G-Cauchy sequence. Since $X$ is G-complete, $\left\{T^{n} x_{0}\right\}$ converges to a point $u \in X$. Now, for $\varepsilon>0$ and by the lower semi-continuity of $\Omega$,

$$
\Omega\left(T^{n} x_{0}, T^{m} x_{0}, u\right) \leq \liminf _{p \rightarrow \infty} \Omega\left(T^{n} x_{0}, T^{m} x_{0}, T^{p} x_{0}\right) \leq \varepsilon, \quad m \geq n
$$


and

$$
\Omega\left(T^{n} x_{0}, u, T^{l} x_{0}\right) \leq \liminf _{p \rightarrow \infty} \Omega\left(T^{n} x_{0}, T^{p} x_{0}, T^{l} x_{0}\right) \leq \varepsilon, \quad l \geq n
$$

Assume that $u \neq T u$. Since $T^{n} x_{0} \leq T^{n+1} x_{0}$,

$$
0<\inf \left\{\Omega\left(T^{n} x_{0}, u, T^{n} x_{0}\right)+\Omega\left(T^{n} x_{0}, u, T^{n+1} x_{0}\right)+\Omega\left(T^{n} x_{0}, T^{n+1} x_{0}, u\right): n \in \mathbb{N}\right\} \leq 3 \varepsilon
$$

a contraction. Hence, we have $u=T u$.

We shall show that $u$ is the unique fixed point of $T$. Suppose, on the contrary, that $v$ is another fixed point of $T$. So, we have

$$
\begin{aligned}
\psi(\Omega(u, u, v)) & =\psi\left(\Omega\left(T u, T^{2} u, T v\right)\right) \\
& \leq \psi(\Omega(u, T u, v))-\phi(\Omega(u, T u, v)) \\
& =\psi(\Omega(u, u, v))-\phi(\Omega(u, u, v)) \\
& <\psi(\Omega(u, u, v))
\end{aligned}
$$

a contraction. Thus, the fixed point $u$ is unique. Now, since $u=T u$, we have

$$
\begin{aligned}
\psi(\Omega(u, u, u)) & =\psi\left(\Omega\left(T u, T^{2} u, T u\right)\right) \\
& \leq \psi(\Omega(u, T u, u))-\phi(\Omega(u, T u, u)) \\
& =\psi(\Omega(u, u, u))-\phi(\Omega(u, u, u)) .
\end{aligned}
$$

So, $\Omega(u, u, u)=0$

Definition 11 Let $(X, \leq)$ be a partially ordered space. Suppose that there exists a $G$-metric on $X$ such that $(X, G)$ is a complete $G$-metric space. A self-mapping $T: X \rightarrow X$ is said to be a weak-contraction mapping if it satisfies the following condition:

$$
\Omega\left(T x, T^{2} x, T y\right) \leq \Omega(x, T x, y)-\phi(\Omega(x, T x, y)) \text { for all } x, y \in X \text {, with } x \leq y,
$$

where $\phi \in \Phi$.

Corollary 12 Let $(X, G, \leq)$ be a partially ordered complete $G$-metric space, and let $\Omega$ be an $\Omega$-distance on $X$. Suppose that a non-decreasing self-mapping $T: X \rightarrow X$ is a weakcontraction mapping, that is,

$$
\Omega\left(T x, T^{2} x, T y\right) \leq \Omega(x, T x, y)-\phi(\Omega(x, T x, y)) \text { for all } x, y \in X, \text { with } x \leq T x
$$

where $\phi \in \Phi$. Suppose also that $\inf \{\Omega(x, y, x)+\Omega(x, y, T x)+\Omega(x, T x, y): x \leq T x\}>0$ for every $y \in X$ with $y \neq T y$. If there exists $x_{0} \in X$ with $x_{0} \leq T x_{0}$, then $T$ has a unique fixed point, say $u \in X$. Moreover, $\Omega(u, u, u)=0$.

If we take $\phi(t)=k t$, where $k \in[0,1)$, we derive Theorem 2.2 [4] as the following corollary. 
Corollary 13 Let $(X, G, \leq)$ be a partially ordered complete G-metric space, and let $\Omega$ be an $\Omega$-distance on $X$. Suppose that there exists $k \in[0,1)$ such that

$$
\Omega\left(T x, T^{2} x, T y\right) \leq k \Omega(x, T x, y) \text { for all } x, y \in X, \text { with } x \leq T x .
$$

Suppose also that $\inf \{\Omega(x, y, x)+\Omega(x, y, T x)+\Omega(x, T x, y): x \leq T x\}>0$ for every $y \in X$ with $y \neq$ Ty. If there exists $x_{0} \in X$ with $x_{0} \leq T x_{0}$, then $T$ has a unique fixed point, say $u \in X$. Moreover, $\Omega(u, u, u)=0$.

Definition 14 Let $(X, \leq)$ be a partially ordered space. Suppose that there exists a $G$-metric on $X$ such that $(X, G)$ is a complete $G$-metric space. A self-mapping $T: X \rightarrow X$ is said to be a Ćirić-type contraction mapping if it satisfies that there exists $0 \leq k<1$ such that

$$
\Omega\left(T x, T^{2} x, T y\right) \leq k M(x, x, y)
$$

where

$$
M(x, x, y)=\max \left\{\Omega(x, T x, T x), \Omega(y, T y, T y), \frac{1}{2} \Omega(x, T y, T y)\right\}
$$

for all $x, y \in X$ with $x \leq y$.

Theorem 15 Let $(X, G, \leq)$ be a partially ordered complete $G$-metric space, and let $\Omega$ be an $\Omega$-distance on $X$. Suppose that a non-decreasing self-mapping $T: X \longrightarrow X$ is a Cirić-type contraction mapping.

(i) For every $x \in X$ and $y \in X$ with $y \neq T(y)$, $\inf \{\Omega(x, y, x)+\Omega(x, y, T x)+\Omega(x, T x, y): x \leq T(x)\}>0$,

(ii) There exists $x_{0} \in X$ such that $x_{0} \leq T\left(x_{0}\right)$,

then $T$ has a fixed point $u$ in $X$ and $\Omega(u, u, u)=0$.

Proof By assumption (ii), there exists $x_{0} \in X$ such that $x_{0} \leq T\left(x_{0}\right)$. We fix $x_{1} \in X$ such that $x_{1}=T\left(x_{0}\right)$. Since $T$ is a non-decreasing mapping, $T x_{0} \leq T x_{1}$. There exists $x_{2} \in X$ such that $T x_{1}=x_{2}$. Recursively, we construct the sequence $\left\{x_{n}\right\}$ in the following way:

$$
x_{n+1}=T x_{n} \leq T x_{n+1}=x_{n+2} \text { for all } n \geq 0 .
$$

Since $T$ is a Cirić-type contraction mapping, by replacing $x=x_{n}$ and $y=x_{n+1}$, we get that

$$
\Omega\left(x_{n+1}, x_{n+2}, x_{n+2}\right)=\Omega\left(T x_{n}, T x_{n+1}, T x_{n+1}\right) \leq k M\left(x_{n}, x_{n}, x_{n+1}\right)
$$

where

$$
\begin{aligned}
M\left(x_{n}, x_{n}, x_{n+1}\right)= & \max \left\{\Omega\left(x_{n}, T x_{n}, T x_{n}\right), \Omega\left(x_{n+1}, T x_{n+1}, T x_{n+1}\right),\right. \\
& \left.\frac{1}{2} \Omega\left(x_{n}, T x_{n+1}, T x_{n+1}\right)\right\} \\
= & \max \left\{\Omega\left(x_{n}, x_{n+1}, x_{n+1}\right), \Omega\left(x_{n+1}, x_{n+2}, x_{n+2}\right),\right.
\end{aligned}
$$




$$
\begin{aligned}
& \left.\frac{1}{2} \Omega\left(x_{n}, x_{n+2}, x_{n+2}\right)\right\} \\
\leq & \max \left\{\Omega\left(x_{n}, x_{n+1}, x_{n+1}\right), \Omega\left(x_{n+1}, x_{n+2}, x_{n+2}\right),\right. \\
& \left.\frac{1}{2}\left[\Omega\left(x_{n}, x_{n+1}, x_{n+1}\right)+\Omega\left(x_{n+1}, x_{n+2}, x_{n+2}\right)\right]\right\} \\
= & \max \left\{\Omega\left(x_{n}, x_{n+1}, x_{n+1}\right), \Omega\left(x_{n+1}, x_{n+2}, x_{n+2}\right)\right\} .
\end{aligned}
$$

Notice that if $M\left(x_{n}, x_{n}, x_{n+1}\right) \leq \Omega\left(x_{n+1}, x_{n+2}, x_{n+2}\right)$, then (2.4) yields a contradiction since $k<1$.

Thus, $M\left(x_{n}, x_{n}, x_{n+1}\right) \leq \Omega\left(x_{n}, x_{n+1}, x_{n+1}\right)$ and inequality (2.4) and $k<1$ turn into

$$
\Omega\left(x_{n+1}, x_{n+2}, x_{n+2}\right) \leq k \Omega\left(x_{n}, x_{n+1}, x_{n+1}\right)
$$

Upon the discussion above, we conclude that the sequence $\left\{\Omega\left(x_{n}, x_{n+1}, x_{n+1}\right)\right\}$ is nonincreasing and bounded below. Therefore, there exists $r \geq 0$ such that

$$
\lim _{n \rightarrow \infty} \Omega\left(x_{n}, x_{n+1}, x_{n+1}\right)=r
$$

We shall show that $r=0$. By a standard calculation, using inequality (2.5) and keeping $k<1$ in mind, we obtain $\lim _{n \rightarrow \infty} \Omega\left(x_{n}, x_{n+1}, x_{n+1}\right)=0$. We claim that the sequence $\left\{x_{n}\right\}$ is G-Cauchy. Let $l \geq m \geq n$ with $m=n+k$ and $l=m+t(k, t \in \mathbb{N})$. By the triangle inequality, we derive that

$$
\begin{aligned}
\Omega\left(x_{n}, x_{m}, x_{l}\right) & \leq \Omega\left(x_{n}, x_{n+1}, x_{n+1}\right)+\Omega\left(x_{n+1}, x_{m}, x_{l}\right) \\
& \leq \Omega\left(x_{n}, x_{n+1}, x_{n+1}\right)+\Omega\left(x_{n+1}, x_{n+2}, x_{n+2}\right)+\cdots+\Omega\left(x_{m-1}, x_{m}, x_{l}\right) .
\end{aligned}
$$

On the other hand, we have

$$
\begin{aligned}
\Omega\left(x_{m-1}, x_{m}, x_{m+t}\right) \leq & k M\left(x_{m-2}, x_{m-2}, x_{m+t-1}\right) \\
= & k \max \left\{\Omega\left(x_{m-2}, x_{m-1}, x_{m-1}\right), \Omega\left(x_{m+t-1}, x_{m+t}, x_{m+t}\right),\right. \\
& \left.\frac{1}{2} \Omega\left(x_{m-2}, x_{m+t}, x_{m+t}\right)\right\} \\
\leq & k \max \left\{\Omega\left(x_{m-2}, x_{m-1}, x_{m-1}\right), \Omega\left(x_{m+t-1}, x_{m+t}, x_{m+t}\right),\right. \\
& \frac{1}{2}\left[\Omega\left(x_{m-2}, x_{m-1}, x_{m-1}\right)+\Omega\left(x_{m-1}, x_{m}, x_{m}\right)\right. \\
& \left.\left.+\cdots+\Omega\left(x_{m+t-1}, x_{m+t}, x_{m+t}\right)\right]\right\} .
\end{aligned}
$$

By combining expressions (2.6) and (2.7), we find that

$$
\begin{aligned}
& \Omega\left(x_{n}, x_{m}, x_{l}\right) \\
& \quad \leq \Omega\left(x_{n}, x_{n+1}, x_{n+1}\right)+\Omega\left(x_{n+1}, x_{n+2}, x_{n+2}\right)+\cdots+\Omega\left(x_{m-2}, x_{m-1}, x_{m-1}\right)
\end{aligned}
$$




$$
\begin{aligned}
& +k \max \left\{\Omega\left(x_{m-2}, x_{m-1}, x_{m-1}\right), \Omega\left(x_{m+t-1}, x_{m+t}, x_{m+t}\right), \frac{1}{2}\left[\Omega\left(x_{m-2}, x_{m-1}, x_{m-1}\right)\right.\right. \\
& \left.\left.+\Omega\left(x_{m-1}, x_{m}, x_{m}\right)+\cdots+\Omega\left(x_{m+t-1}, x_{m+t}, x_{m+t}\right)\right]\right\} .
\end{aligned}
$$

Taking $n \rightarrow \infty$ in (2.8), we conclude that

$$
\lim _{n, m, l \rightarrow \infty} \Omega\left(x_{n}, x_{m}, x_{l}\right)=0
$$

and hence $\left\{x_{n}\right\}$ is a $G$-Cauchy sequence due to expression (c) of Lemma 6. Since $X$ is $G$ complete, $\left\{x_{n}\right\}$ converges to a point $u \in X$. Thus, for $\varepsilon>0$ and by the lower semi-continuity of $\Omega$, we have

$$
\Omega\left(x_{n}, x_{m}, u\right) \leq \liminf _{p \rightarrow \infty} \Omega\left(x_{n}, x_{m}, x_{p}\right) \leq \varepsilon, \quad m \geq n,
$$

and

$$
\Omega\left(x_{n}, u, x_{l}\right) \leq \liminf _{p \rightarrow \infty} \Omega\left(x_{n}, x_{p}, x_{l}\right) \leq \varepsilon, \quad l \geq n
$$

Assume that $u \neq T u$. Since $x_{n+1} \leq x_{n+2}$,

$$
0<\inf \left\{\Omega\left(x_{n+1}, u, x_{n+1}\right)+\Omega\left(x_{n+1}, u, x_{n+2}\right)+\Omega\left(x_{n+1}, x_{n+2}, u\right): n \in \mathbb{N}\right\} \leq 3 \varepsilon
$$

for every $\varepsilon>0$, that is a contraction. Therefore, we have $u=T u$ and $\Omega(u, u, u)=0$.

Definition 16 Let $(X, \leq)$ be a partially ordered space and $f, g: X \rightarrow X$. We say that $g$ is an $f$-monotone mapping if

$$
x, y \in X, \quad f(x) \leq f(y) \quad \Longrightarrow \quad g(x) \leq g(y) .
$$

Theorem 17 Let $(X, G, \leq)$ be a partially ordered complete $G$-metric space, and let $\Omega$ be an $\Omega$-distance on $X$ such that $X$ is $\Omega$-bounded. Let $f: X \rightarrow X$ and $g: f(X) \longrightarrow X$ commute, $f$ be non-decreasing and $g$ be an $f$-monotone mapping such that:

(a) $g f(X) \subseteq f^{2}(X)$;

(b) $\Omega\left(g f x, g y, g^{2} x\right) \leq k M(x, x, y)$, where $M(x, x, y)=\max \left\{\Omega\left(f^{2} x, f y, f g x\right), \Omega(f y, f y, g y), \Omega\left(f^{2} x, f^{2} x, f g x\right)\right\}$ for all $x, y \in X$ with $f(x) \leq f(y)$ and $0 \leq k<1$;

(c) for every $x \in X$ and $z \in X$ with $f^{2} z \neq g f z$,

$$
\inf \left\{\Omega(x, z, x)+\Omega(x, x, z)+\Omega\left(f^{2} x, g x, g f x\right): f^{2} x \leq g f x\right\}>0
$$

(d) there exists $x_{0} \in f(X)$ such that $f\left(x_{0}\right) \leq g\left(x_{0}\right)$;

then $f$ and $g$ have a unique common fixed point $u$ in $X$ and $\Omega(u, u, u)=0$.

Proof Let $x_{0} \in f(X)$ such that $f\left(x_{0}\right) \leq g\left(x_{0}\right)$. By part (a), we can choose $x_{1} \in f(X)$ such that $f\left(x_{1}\right)=g\left(x_{0}\right)$. Again from part (a), we can choose $x_{2} \in f(X)$ such that $f\left(x_{2}\right)=g\left(x_{1}\right)$. 
Continuing this process, we can construct sequences $\left\{x_{n}\right\}$ in $f(X)$ and $\left\{z_{n}\right\}$ in $f^{2}(X)$ such that

$$
y_{n}=g x_{n}=f x_{n+1},
$$

and

$$
z_{n}=g y_{n-1}=g f x_{n}=f g x_{n}=f y_{n}
$$

Since $f\left(x_{0}\right) \leq g\left(x_{0}\right)$ and $f\left(x_{1}\right)=g\left(x_{0}\right)$, we have $f\left(x_{0}\right) \leq f\left(x_{1}\right)$. Then by Definition $16, g\left(x_{0}\right) \leq$ $g\left(x_{1}\right)$. Continuing, we obtain

$$
g x_{n} \leq g x_{n+1}, \quad \forall n \geq 0
$$

So, by (2.9) and (2.11), for all $t \geq 1, f x_{n} \leq f x_{n+t}$. Now, for all $s \geq 0$,

$$
\begin{aligned}
\Omega\left(z_{n}, z_{n+s}, z_{n+1}\right)= & \Omega\left(g f x_{n}, g x_{n+s-1}, g^{2} x_{n}\right) \\
\leq & k \max \left\{\Omega\left(f^{2} x_{n}, f y_{n+s-1}, f g x_{n}\right), \Omega\left(f y_{n+s-1}, f y_{n+s-1}, g y_{n+s-1}\right),\right. \\
& \left.\Omega\left(f^{2} x_{n}, f^{2} x_{n}, f g x_{n}\right)\right\} \\
= & k \max \left\{\Omega\left(z_{n-1}, z_{n+s-1}, z_{n}\right), \Omega\left(z_{n+s-1}, z_{n+s-1}, z_{n+s}\right),\right. \\
& \left.\Omega\left(z_{n-1}, z_{n-1}, z_{n}\right)\right\} .
\end{aligned}
$$

Then, for $s=0$,

$$
\Omega\left(z_{n}, z_{n}, z_{n+1}\right) \leq k \Omega\left(z_{n-1}, z_{n-1}, z_{n}\right)
$$

For $s=1$,

$$
\Omega\left(z_{n}, z_{n+1}, z_{n+1}\right) \leq k^{1+1} \max \left\{\Omega\left(z_{n-1}, z_{n}, z_{n}\right), \Omega\left(z_{n-1}, z_{n-1}, z_{n}\right)\right\} .
$$

For $s=2$,

$$
\Omega\left(z_{n}, z_{n+2}, z_{n+1}\right) \leq k^{1+2} \max \left\{\Omega\left(z_{n-1}, z_{n+1}, z_{n}\right), \Omega\left(z_{n-1}, z_{n-1}, z_{n}\right)\right\}
$$

and

$$
\begin{aligned}
\Omega\left(z_{n-1}, z_{n-1}, z_{n}\right) & \leq k \max \left\{\Omega\left(z_{n-2}, z_{n-2}, z_{n-1}\right), \Omega\left(z_{n-2}, z_{n-2}, z_{n-1}\right), \Omega\left(z_{n-2}, z_{n-2}, z_{n-1}\right)\right\} \\
& =k \Omega\left(z_{n-2}, z_{n-2}, z_{n-1}\right) \\
& \vdots \\
& \leq k^{n-1} \Omega\left(z_{0}, z_{0}, z_{1}\right) .
\end{aligned}
$$

Therefore, for all $n \geq 1$ and $s \geq 0$,

$$
\Omega\left(z_{n}, z_{n+s}, z_{n+1}\right) \leq k^{n+s} \max \left\{\Omega\left(z_{n-1}, z_{n+s-1}, z_{n}\right), \Omega\left(z_{0}, z_{0}, z_{1}\right)\right\}
$$


Notice that if $\Omega\left(z_{n}, z_{n+s}, z_{n+1}\right) \leq k^{n+s} \Omega\left(z_{0}, z_{0}, z_{1}\right)$, so for all $s \geq 0, \lim _{n \rightarrow \infty} \Omega\left(z_{n}, z_{n+s}, z_{n+1}\right)=$ 0 . If $\Omega\left(z_{n}, z_{n+s}, z_{n+1}\right) \leq k^{n+s} \Omega\left(z_{n-1}, z_{n+s-1}, z_{n}\right)$, so $\left\{\Omega\left(z_{n-1}, z_{n+s-1}, z_{n}\right)\right\}$ is non-increasing and bounded below. Therefore, there exists $r \geq 0$ such that

$$
\lim _{n \rightarrow \infty} \Omega\left(z_{n-1}, z_{n+s-1}, z_{n}\right)=r
$$

We shall show that $r=0$. By a standard calculation, using inequality (2.12) and keeping $k<$ 1 in mind, we obtain $\lim _{n \rightarrow \infty} \Omega\left(z_{n-1}, z_{n+s-1}, z_{n}\right)=0$. Now, for any $l \geq m \geq n$ with $m=n+k$ and $l=m+t(k, t \in \mathbb{N})$, we have

$$
\begin{aligned}
\Omega\left(z_{n}, z_{m}, z_{l}\right) \leq & \Omega\left(z_{n}, z_{n+1}, z_{n+1}\right)+\Omega\left(z_{n+1}, z_{m}, z_{l}\right) \\
\leq & \Omega\left(z_{n}, z_{n+1}, z_{n+1}\right)+\Omega\left(z_{n+1}, z_{n+2}, z_{n+2}\right)+\cdots+\Omega\left(z_{m-1}, z_{m}, z_{l}\right) \\
\leq & \Omega\left(z_{n}, z_{n+1}, z_{n+1}\right)+\Omega\left(z_{n+1}, z_{n+2}, z_{n+2}\right)+\cdots+\Omega\left(z_{m-1}, z_{m}, z_{m}\right) \\
& +\Omega\left(z_{m}, z_{m+1}, z_{m+1}\right)+\cdots+\Omega\left(z_{m+t-1}, z_{m}, z_{m+t}\right) .
\end{aligned}
$$

So,

$$
\lim _{n, m, l \rightarrow \infty} \Omega\left(z_{n}, z_{m}, z_{l}\right)=0
$$

and consequently, by Part (3) of Lemma $6,\left\{z_{n}\right\}$ is a G-Cauchy sequence. Since $X$ is Gcomplete, $\left\{z_{n}\right\}$ converges to a point $z \in X$. Thus, for $\varepsilon>0$ and by the lower semi-continuity of $\Omega$, we have

$$
\Omega\left(z_{n}, z_{m}, z\right) \leq \liminf _{p \rightarrow \infty} \Omega\left(z_{n}, z_{m}, z_{p}\right) \leq \varepsilon, \quad m \geq n,
$$

and

$$
\Omega\left(z_{n}, z, z_{l}\right) \leq \liminf _{p \rightarrow \infty} \Omega\left(z_{n}, z_{p}, z_{l}\right) \leq \varepsilon, \quad l \geq n
$$

Assume that $f^{2} z \neq g f z$. Since $f$ is non-decreasing, we obtain

$$
z_{n}=f^{2} x_{n+1}=f\left(f x_{n+1}\right) \leq f\left(f x_{n+2}\right)=g f x_{n+1}=z_{n+1},
$$

then $z_{n} \leq z_{n+1}$. Also, for all $n \geq 1$,

$$
\begin{aligned}
\Omega\left(f^{2} z_{n}, g z_{n}, g f z_{n}\right)= & \Omega\left(g f z_{n-1}, g z_{n}, g^{2} z_{n-1}\right) \\
\leq & k \max \left\{\Omega\left(f^{2} z_{n-1}, f z_{n}, f g z_{n-1}\right), \Omega\left(f z_{n}, f z_{n}, g z_{n}\right),\right. \\
& \left.\Omega\left(f^{2} z_{n-1}, f^{2} z_{n-1}, f g z_{n-1}\right)\right\} \\
= & k \max \left\{\Omega\left(g f z_{n-2}, g z_{n-1}, g^{2} z_{n-2}\right), \Omega\left(f z_{n}, f z_{n}, g z_{n}\right),\right. \\
& \left.\Omega\left(g f z_{n-2}, g f z_{n-2}, g^{2} z_{n-2}\right)\right\} \\
\leq & k^{3} \max \left\{\Omega\left(f^{2} z_{n-2}, f z_{n-1}, f g z_{n-2}\right), \Omega\left(f z_{n-1}, f z_{n-1}, g z_{n-1}\right),\right. \\
& \Omega\left(f^{2} z_{n-2}, f^{2} z_{n-2}, f g z_{n-2}\right), \Omega\left(f z_{n}, f z_{n}, g z_{n}\right),
\end{aligned}
$$




$$
\begin{aligned}
& \Omega\left(f^{2} z_{n-2}, f^{2} z_{n-2}, f g z_{n-2}\right), \Omega\left(f^{2} z_{n-2}, f^{2} z_{n-2}, g f z_{n-2}\right), \\
& \left.\Omega\left(f^{2} z_{n-2}, f^{2} z_{n-2}, f g z_{n-2}\right)\right\} \\
= & k^{3} \max \left\{\Omega\left(f^{2} z_{n-2}, f z_{n-1}, f g z_{n-2}\right), \Omega\left(f z_{n-1}, f z_{n-1}, g z_{n-1}\right),\right. \\
& \left.\Omega\left(f^{2} z_{n-2}, f^{2} z_{n-2}, f g z_{n-2}\right), \Omega\left(f z_{n}, f z_{n}, g z_{n}\right)\right\} \\
\vdots & \\
\leq & k^{2 n+1} \max \left\{\Omega\left(f^{2} z_{1}, g z_{1}, g f z_{1}\right), \Omega\left(f^{2} z_{1}, f^{2} z_{1}, f g z_{1}\right),\right. \\
& \left.\Omega\left(f z_{i}, f z_{i}, g z_{i}\right), 0 \leq i \leq n\right\} \\
\leq & k^{2 n+1} C
\end{aligned}
$$

where $C=\max \left\{\Omega\left(f^{2} z_{1}, g z_{1}, g f z_{1}\right), \Omega\left(f^{2} z_{1}, f^{2} z_{1}, f g z_{1}\right), \Omega\left(f z_{i}, f z_{i}, g z_{i}\right), 0 \leq i \leq n\right\}$, and consequently $\lim _{n \rightarrow \infty} \Omega\left(f^{2} z_{n}, g z_{n}, g f z_{n}\right)=0$. Therefore,

$$
0<\inf \left\{\Omega\left(z_{n}, z, z_{n}\right)+\Omega\left(z_{n}, z_{n}, z\right)+\Omega\left(f^{2} z_{n}, g z_{n}, g f z_{n}\right): n \in \mathbb{N}\right\} \leq 3 \varepsilon
$$

for every $\varepsilon>0$, that is a contraction. So, we have $f^{2} z=g f z$. Then, by (b),

$$
\begin{aligned}
\Omega\left(g f^{2} z, g(g f z), g^{2} f z\right) \leq & k \max \left\{\Omega\left(f^{2} f z, f(g f z), f g(f z)\right), \Omega(f(g f z), f(g f z), g(g f z)),\right. \\
& \left.\Omega\left(f^{2}(f z), f^{2}(f z), f g(f z)\right)\right\} .
\end{aligned}
$$

So, $\Omega\left(g f^{2} z, g(g f z), g^{2} f z\right)=0$. Since $X$ is $\Omega$-bounded, $\Omega\left(g f^{2} z, g(g f z), g^{2} f z\right)=0<M$. Similarly, $\Omega\left(g f^{2} z, g f z, g^{2} f z\right) \leq k \Omega\left(f^{2} z, f^{2} z, f^{2} z\right)<M$. By part (c) of Definition 3, G(gf $\left.z, g f z, g^{2} f z\right)=0$. Then $g^{2} f z=g f z$, which implies that $g f z$ is a fixed point for $g$. Now,

$$
f(g f z)=g f^{2} z=g^{2} f z=g f z
$$

Then $u=g f z$ is a common fixed point of $f$ and $g$.

Uniqueness. Assume that there exists $v \in X$ such that $f v=g v=v$. Hence, we have

$$
\Omega(v, v, v) \leq k \Omega(v, v, v),
$$

and so $\Omega(v, v, v)=\Omega(u, u, u)=0$. Also, $\Omega(v, u, v)=0$. Then, by Part (c) of Definition 3, $u=v$ and $\Omega(u, u, u)=0$.

The following corollary is a generalization of Theorem 2.1 [14].

Denote by $\Lambda$ the set of all functions $\lambda:[0,+\infty) \rightarrow[0,+\infty)$ satisfying the following hypotheses:

(i) $\lambda$ is a Lebesgue-integrable mapping on each compact subset of $[0,+\infty)$,

(ii) for every $\varepsilon>0$, we have $\int_{0}^{\varepsilon} \lambda(s) d s>0$,

(iii) $\|\lambda\|<1$, where $\|\lambda\|$ denotes the norm of $\lambda$.

Now, we have the following corollary.

Corollary 18 Let $(X, G, \leq)$ be a partially ordered complete G-metric space, let $\Omega$ be an $\Omega$ distance on $X$, and let $T: X \rightarrow X$ be a non-decreasing self-mapping. Suppose that $\psi \in \Psi$ 
and $\phi \in \Phi$ such that

$$
\int_{0}^{\psi\left(\Omega\left(T x, T^{2} x, T y\right)\right)} \lambda(s) d s \leq \int_{0}^{\psi(\Omega(x, T x, y))} \lambda(s) d s-\int_{0}^{\phi(\Omega(x, T x, y))} \lambda(s) d s
$$

for all $x \leq T x, y \in X$, where $\lambda \in \Lambda$. Also, for every $x \in X$,

$$
\inf \{\Omega(x, y, x)+\Omega(x, y, T x)+\Omega(x, T x, y): x \leq T x\}>0
$$

for every $y \in X$ with $y \neq T y$. If there exists $x_{0} \in X$ with $x_{0} \leq T x_{0}$, then $T$ has a unique fixed point.

Proof Define $\gamma:[0,+\infty) \rightarrow[0,+\infty)$ by $\gamma(t)=\int_{0}^{t} \lambda(s) d s$, then from inequality (2.13), we have

$$
\gamma\left(\psi\left(\Omega\left(T x, T^{2} x, T y\right)\right)\right) \leq \gamma(\psi(\Omega(x, T x, y)))-\gamma(\phi(\Omega(x, T x, y)))
$$

which can be written as

$$
\psi_{1}\left(\Omega\left(T x, T^{2} x, T y\right)\right) \leq \psi_{1}(\Omega(x, T x, y))-\phi_{1}(\Omega(x, T x, y)),
$$

where $\psi_{1}=\gamma \circ \psi$ and $\phi_{1}=\gamma \circ \phi$. Since the functions $\psi_{1}$ and $\phi_{1}$ satisfy the properties of $\psi$ and $\phi$, by Theorem 10, $T$ has a unique fixed point.

Corollary 19 Let $(X, G, \leq)$ be a partially ordered complete G-metric space, let $\Omega$ be an $\Omega$-distance on $X$, and let $T: X \rightarrow X$ be a non-decreasing self-mapping. Suppose that there exists $0 \leq k<1$ such that

$$
\int_{0}^{\psi\left(\Omega\left(T x, T^{2} x, T y\right)\right)} k \lambda(s) d s \leq \int_{0}^{M(x, x, y)} \lambda(s) d s
$$

for all $x \leq T x, y \in X$, where

$$
M(x, x, y)=\max \left\{\Omega(x, T x, T x), \Omega(y, T y, T y), \frac{1}{2} \Omega(x, T y, T y)\right\}
$$

and $\lambda \in \Lambda$. Also, for every $x \in X$,

$$
\inf \{\Omega(x, y, x)+\Omega(x, y, T x)+\Omega(x, T x, y): x \leq T x\}>0
$$

for every $y \in X$ with $y \neq T y$. If there exists $x_{0} \in X$ with $x_{0} \leq T x_{0}$, then $T$ has a unique fixed point.

\section{Application}

In this section, we give an existence theorem for a solution of the following integral equations:

$$
x(t)=\int_{0}^{1} K(t, s, x(s)) d s+g(t), \quad t \in[0,1] .
$$


Let $X=C([0,1])$ be the set of all continuous functions defined on $[0,1]$. Define $G: X \times$ $X \times X \rightarrow \mathbb{R}$ by

$$
G(x, y, z)=\|x-y\|+\|y-z\|+\|z-x\|,
$$

where $\|x\|=\sup \{|x(t)|: t \in[0,1]\}$. Then $(X, G)$ is a complete $G$-metric space. Let $\Omega=G$. Then $\Omega$ is an $\Omega$-distance on $X$. Define an ordered relation $\leq$ on $X$ by

$$
x \leq y \quad \text { iff } \quad x(t) \leq y(t), \quad \forall t \in[0,1] .
$$

Then $(X, \leq)$ is a partially ordered set. Now, we prove the following result.

Theorem 20 Suppose the following hypotheses hold:

(1) $K:[0,1] \times[0,1] \times \mathbb{R}^{+} \rightarrow \mathbb{R}^{+}$and $g:[0,1] \rightarrow \mathbb{R}$ are continuous mappings,

(2) $K$ is non-decreasing in its first coordinate and $g$ is non-decreasing,

(3) There exists a continuous function $G:[0,1] \times[0,1] \rightarrow[0,+\infty)$ such that

$$
|K(t, s, u)-K(t, s, v)| \leq G(t, s)|u-v|
$$

for every comparable $u, v \in \mathbb{R}^{+}$and $s, t \in[0,1]$ with $\sup _{t \in[0,1]} \int_{0}^{1} G(t, s) d s \leq \frac{1}{2}$,

(4) There exist continuous, non-decreasing functions $\phi, \psi:[0, \infty) \rightarrow(0, \infty)$ with $\psi^{-1}(\{0\})=\phi^{-1}(\{0\})=\{0\}$ and $\psi(r) \leq \psi(2 r)-\phi(2 r)$ for all $r \in[0, \infty)$.

Then the integral equation has a solution in $C([0,1])$.

Proof Define $T x(t)=\int_{0}^{1} K(t, s, x(s)) d s+g(t)$. By hypothesis (2), we have that $T$ is nondecreasing.

Now, if

$$
\inf \{\Omega(x, y, x)+\Omega(x, y, T x)+\Omega(x, T x, y): x \leq T x\}=0
$$

for every $y \in X$ with $y \neq T y$, then for each $n \in \mathbb{N}$, there exists $x_{n} \in C([0,1])$ with $x_{n} \leq T x_{n}$ such that

$$
\Omega\left(x_{n}, y, x_{n}\right)+\Omega\left(x_{n}, y, T x_{n}\right)+\Omega\left(x_{n}, T x_{n}, y\right) \leq \frac{1}{n} .
$$

Then we have

$$
\Omega\left(x_{n}, y, T x_{n}\right)=\sup _{t \in[0,1]}\left|x_{n}-y\right|+\sup _{t \in[0,1]}\left|y-T x_{n}\right|+\sup _{t \in[0,1]}\left|T x_{n}-x_{n}\right| \leq \frac{1}{n} .
$$

Thus,

$$
\lim _{n \rightarrow \infty} x_{n}(t)=y(t), \quad \lim _{n \rightarrow \infty} T x_{n}(t)=y(t) .
$$

By the continuity of $K$, we have

$$
\begin{aligned}
y(t) & =\lim _{n \rightarrow \infty} T x_{n}(t)=\int_{0}^{1} K\left(t, s, \lim _{n \rightarrow \infty} x_{n}(s)\right) d s+g(t) \\
& =\int_{0}^{1} K(t, s, y(s)) d s+g(t)=T y(t),
\end{aligned}
$$


which is a contradiction. Therefore,

$$
\inf \{\Omega(x, y, x)+\Omega(x, y, T x)+\Omega(x, T x, y): x \leq T x\}>0 .
$$

Now, for $x, y \in X$ with $x \leq T x$, we have

$$
\begin{aligned}
\psi\left(\Omega\left(T x, T^{2} x, T y\right)\right)= & \psi\left(\sup _{t \in[0,1]}\left|T x(t)-T^{2} x(t)\right|+\sup _{t \in[0,1]}\left|T^{2} x(t)-T y(t)\right|\right. \\
& \left.+\sup _{t \in[0,1]}|T y(t)-T x(t)|\right) \\
\leq & \psi\left(\sup _{t \in[0,1]} \int_{0}^{1}|K(t, s, x(s))-K(t, s, T x(s))| d s\right. \\
& +\sup _{t \in[0,1]} \int_{0}^{1}|K(t, s, T x(s))-K(t, s, y(s))| d s \\
& \left.+\sup _{t \in[0,1]} \int_{0}^{1}|K(t, s, y(s))-K(t, s, x(s))| d s\right) \\
\leq & \psi\left(\sup _{t \in[0,1]}\left(\int_{0}^{1} G(t, s)|x(s)-T x(s)| d s\right)\right. \\
& +\sup _{t \in[0,1]}\left(\int_{0}^{1} G(t, s)|T x(s)-y(s)| d s\right) \\
& \left.+\sup _{t \in[0,1]}\left(\int_{0}^{1} G(t, s)|y(s)-x(s)| d s\right)\right) \\
\leq & \psi\left(\sup _{t \in[0,1]}(|x(t)-T x(t)|) \sup _{t \in[0,1]} \int_{0}^{1} G(t, s) d s\right. \\
& +\sup _{t \in[0,1]}(|T x(t)-y(t)|) \sup _{t \in[0,1]} \int_{0}^{1} G(t, s) d s \\
& \left.+\sup _{t \in[0,1]}(|y(t)-x(t)|) \sup _{t \in[0,1]} \int_{0}^{1} G(t, s) d s\right) \\
\leq & \psi\left(\frac{1}{2} \sup _{t \in[0,1]}(|x(t)-T x(t)|)+\frac{1}{2} \sup _{t \in[0,1]}(|T x(t)-y(t)|)\right. \\
& \left.+\frac{1}{2} \sup _{t \in[0,1]}(|y(t)-x(t)|)\right) \\
\leq & \left(\frac{1}{2} \Omega(x, T x, y)\right) \leq \psi(\Omega(x, T x, y))-\phi(\Omega(x, T x, y)) . \\
&
\end{aligned}
$$

Thus, by Theorem 10, there exists a solution $u \in C[0,1]$ of integral equation (3.1). 


\section{Author details}

'Department of Mathematics, Islamic Azad University, Sari Branch, Sari, Iran. ${ }^{2}$ Department of Mathematics, Atilim University, İncek, Ankara 06836, Turkey.

\section{Acknowledgements}

The authors thank anonymous reviewers for their remarkable comments, suggestions and ideas that helped to improve this paper.

\section{Received: 19 July 2013 Accepted: 7 October 2013 Published:\#PUBLICATION_DATE}

\section{References}

1. Jleli, M, Samet, B: Remarks on G-metric spaces and fixed point theorems. Fixed Point Theory Appl. 2012, 210 (2012)

2. Samet, B, Vetro, C, Vetro, F: Remarks on G-metric spaces. Int. J. Anal. 2013, Article ID 917158 (2013)

3. Mustafa, Z, Sims, B: A new approach to generalized metric spaces. J. Nonlinear Convex Anal. 7, $289-297$ (2006)

4. Saadati, R, Vaezpour, SM, Vetro, P, Rhoades, BE: Fixed point theorems in generalized partially ordered G-metric spaces. Math. Comput. 52, 797-801 (2010)

5. Kada, O, Suzuki, T, Takahashi, W: Nonconvex minimization theorems and fixed point theorems in complete metric space. Math. Jpn. 44, 381-391 (1996)

6. Gholizadeh, L, Saadati, R, Shatanawi, W, Vaezpour, SM: Contractive mapping in generalized, ordered metric spaces with application in integral equations. Math. Probl. Eng. 2011, Article ID 380784 (2011)

7. Gholizadeh, L: A fixed point theorem in generalized ordered metric spaces with application. J. Nonlinear Sci. Appl. 6, 244-251 (2013)

8. Abbas, M, Rhoades, B: Common fixed point results for non-commuting mappings without continuity in generalized metric spaces. Appl. Math. Comput. 215, 262-269 (2009)

9. Karapinar, E, Agarwal, RP: Further fixed point results on G-metric spaces. Fixed Point Theory Appl. 2013, 154 (2013)

10. Asadi, M, Karapinar, E, Salimi, P: A new approach to G-metric and related fixed point theorems. J. Inequal. Appl. (2013)

11. Agarwal, R, Karapınar, E: Remarks on some coupled fixed point theorems in G-metric spaces. Fixed Point Theory Appl. 2013, 2 (2013)

12. Gnana Bhaskar, T, Lakshmikantham, V: Fixed point theorems in partially ordered metric spaces and applications. Nonlinear Anal. TMA 65, 1379-1393 (2006)

13. Ran, ACM, Reurings, MCB: A fixed point theorem in partially ordered sets and some applications to matrix equations. Proc. Am. Math. Soc. 132, 1435-1443 (2004)

14. Manro, S, Bhatia, SS, Kumar, S: Expansion mappings theorems in G-metric spaces. Int. J. Contemp. Math. Sci. 5(51), 2529-2535 (2010)

\#DIGITAL_OBJECT_IDENTIFIER

Cite this article as: Gholizadeh and Karapınar: Remarks on contractive mappings via $\Omega$-distance. Journal of Inequalities and Applications \#CITATION

\section{Submit your manuscript to a SpringerOpen ${ }^{\circ}$ journal and benefit from:}

- Convenient online submission

- Rigorous peer review

- Immediate publication on acceptance

- Open access: articles freely available online

- High visibility within the field

- Retaining the copyright to your article 